\title{
Aproximación a la cronología de la pesca en el bajo de Sarmiento (Chubut, Argentina) a partir de la distribución de pesos líticos
}

\section{Julián E. Moreno}

Facultad de Humanidades y Ciencias Sociales, Universidad de la Patagonia "San Juan Bosco" (UNPSJB) - Instituto de Diversidad y Evolución Austral (IDEAus), Centro Nacional Patagónico (CENPAT) y Consejo Nacional de Investigaciones Científicas y Técnicas (CONICET). Boulevard Brown 2915 (CP U9120ACD), Puerto Madryn, Chubut, Argentina. E-mail: julianemoreno@yahoo.com

\section{Santiago Peralta González}

Facultad de Humanidades y Ciencias Sociales, Universidad de la Patagonia "San Juan Bosco" (UNPSJB) - Instituto de Diversidad y Evolución Austral (IDEAus), Centro Nacional Patagónico (CENPAT) y Consejo Nacional de Investigaciones Científicas y Técnicas (CONICET). Boulevard Brown 2915 (CP U9120ACD), Puerto Madryn, Chubut, Argentina. E-mail: peraltagonzalezsantiago@gmail.com

\section{Ariadna Svoboda}

\section{(iD https://orcid.org/0000-0003-4628-5454}

Instituto de Diversidad y Evolución Austral (IDEAus), Centro Nacional Patagónico (CENPAT) y Consejo Nacional de Investigaciones Científicas y Técnicas (CONICET). Boulevard Brown 2915 (CP U9120ACD), Puerto Madryn, Chubut, Argentina. E-mail: ariadnasvoboda@hotmail.com

Recibido: 31 de marzo de 2020 Aceptado: 29 de septiembre de 2020

\begin{abstract}
Resumen
Las investigaciones arqueológicas en el bajo de Sarmiento (centro-sur de la provincia del Chubut) permitieron argumentar que la pesca fue una actividad importante entre las poblaciones cazadoras-recolectoras. La información disponible nos permite ubicar el inicio de dicha actividad aproximadamente en 1500 años AP. En este trabajo se evalúa la distribución de los pesos líticos (plomadas de redes) como indicadores de la cronología de la actividad pesquera, tomando como marco de referencia el esquema de evolución del paleolago que cubrió la totalidad del bajo. Los resultados indican una mayor frecuencia de hallazgos de pesos líticos en los sectores del fondo de valle, disponible para la ocupación humana luego del Holoceno tardío final, lo que sugiere que la pesca es un fenómeno posterior al 1500 AP. Se incluyen en la discusión problemas de visibilidad, coleccionismo y tafonómicos, además de datos arqueofaunísticos, cronológicos y tecnológicos.
\end{abstract}




\title{
Chronological approach to fishing at Sarmiento Basin (Chubut, Argentina) through lithic weights distribution
}

\begin{abstract}
Archaeological investigations at Sarmiento basin (south-central Chubut province) allowed arguing that fishing was an important activity among hunter-gatherer populations. Available information places this activity at least at 1500 years BP. Using the evolution of a paleolake that covered the entire basin as a reference, this paper considers the distribution of lithic weights (net sinkers) as an indicator of fishing chronology. Results indicate a higher frequency of lithic weights in the lower sectors of the valley, which was available for human occupation after the Late Holocene, suggesting that fishing activity might be assigned to a period after 1500 years BP. Visibility, collecting, and taphonomic problems are included in the discussion in addition to archaeofaunal, chronological, and technological data.
\end{abstract}

KEYWORDS: Subsistence; Paleolake; Lacustrine environment; Hunters-gatherers; Central Patagonia.

\section{Introducción}

Se ha postulado que la subsistencia de las antiguas poblaciones cazadoras-recolectoras del bajo de Sarmiento (centro-sur de la provincia de Chubut) incluyó un aprovechamiento importante de peces lacustres. En el lago Musters los peces representan el 25\% (en NISP) de la fauna, mientras que en el sitio Delta del Arroyo Vulcana 1 y en el sitio Laguna de la Flecha 10 alcanzan el $20 \%$. En los sitios del vecino lago Colhué Huapi estas cifras suben al 94\% en Valle Hermoso 4 y al 99\% en Boliche de Jerez 3 (Moreno, et al., 2015; Reyes y Svoboda, 2016; Svoboda y Moreno, 2018). Como parte de esta estrategia, los grupos incorporaron tecnologías especializadas en la obtención de estos recursos tales como redes de pesca $y$, en menor medida, artefactos de pesca individual ( $p$. ej. líneas y arpones). Tomando las modalidades de uso de los ambientes acuáticos definidas por Prates y Bonomo (2017), las estrategias identificadas adoptadas por los cazadores-recolectores del bajo de Sarmiento responden a un concepto intermedio entre las categorías de uso sostenido y uso intensivo y sistemático. Es decir que los peces lacustres tuvieron una importancia significativa pero no necesariamente central en la subsistencia; no obstante, disponían de una tecnológica específica para la captura de estas presas (redes, líneas, arpones). Por su parte, aún se encuentran en estudio algunas líneas de evidencia (por ejemplo, lectura de bandas de crecimiento en otolitos y escamas de perca) que ayuden a comprender las modalidades de uso estacional o anual de los peces lacustres.

Si bien el área de estudio ha sido ocupada por cazadores-recolectores desde el Holoceno medio -al menos 5000 años AP (Moreno et al., 2015) - la temporalidad del aprovechamiento de peces sólo pudo ser asignada, por el momento, al Holoceno tardío final, luego del 1500 AP (Svoboda y Moreno, 2018). Esta inferencia fue derivada de estudios zooarqueológicos realizados en sitios con cronología posterior a 1500 $A P$, que presentan una abrumadora frecuencia de restos de peces lacustres (perca y pejerrey patagónico) en sus depósitos (Svoboda, 2019; Svoboda y Moreno, 2018). Por el contrario, la ausencia de restos faunísticos en los depósitos datados entre 5000 a $1500 \mathrm{AP}$ no ha permitido evaluar tendencias en la subsistencia ni la antigüedad del uso de peces. No obstante, en un trabajo anterior se planteó la posibilidad de que la actividad de pesca fuera más antigua debido al hallazgo de pesos líticos en dos sitios superficiales ubicados entre 12 y $32 \mathrm{~km}$ respectivamente de la costa actual de los 
lagos, interpretándose que podrían asociarse a niveles más elevados del paleolago (Reyes y Svoboda, 2016).

En este marco, en el presente trabajo se utilizan los pesos líticos como indicadores indirectos del aprovechamiento de peces lacustres. Estos artefactos son los componentes perdurables de los sistemas de pesca y se definen como guijarros con escotaduras piqueteadas confeccionadas con un canto rodado, un clasto anguloso, una laja formatizada u otra forma base sobre la cual, por piqueteado, se produjeron dos escotaduras o comienzos de surco en un extremo opuesto (Orquera y Piana, 1986 en Torres, 2007). En el bajo de Sarmiento hay una alta frecuencia de pesos líticos en contextos superficiales cuya función es atribuible a las actividades de obtención de peces (Reyes y Svoboda, 2016).

El objetivo entonces es evaluar la distribución de pesos líticos en referencia al paleolago del bajo de Sarmiento, el cual cubrió toda su superficie y comenzó su descenso a lo largo del Holoceno (Moreno, Pérez Ruiz y Ramírez Rozzi, 2016). La hipótesis que organiza el trabajo supone que la pesca se acota al Holoceno tardío final, y la expectativa es que los pesos líticos se concentren en el sector más bajo de la cuenca por lo que su antigüedad máxima sería de 1500 años AP, como se desarrolla a continuación. En esta exposición se presentan además nuevas dataciones de otros sectores del lago que complementan a los publicados con anterioridad y arrojan información sobre la evolución del paleolago.

\section{La evolución del paleolago del bajo de Sarmiento}

El bajo de Sarmiento se ubica en el centro-sur de la provincia del Chubut y consiste en una depresión de 4200 ha, con el eje mayor en sentido E-O de 70 km y 60 km N-S. Está limitado al oeste por la Sierra de San Bernardo, al sur y este por la Meseta del Castillo y al norte la Sierra Corrientes y la Pampa de los Guanacos (Figura 1).

En el bajo se pueden distinguir dos grandes unidades de paisaje: laderas relativamente abruptas que descienden alrededor de $100 \mathrm{~m}$ en relación al terreno circundante y un extenso fondo de valle (entre 20 y $50 \mathrm{~km}$ ) de escasa pendiente, en donde se encuentran los lagos Musters y Colhué Huapi junto a sus respectivas planicies de inundación actuales (Figura 2). Se considera como ladera todo el paisaje comprendido entre los 280 m s.n.m. (10 m sobre el nivel del lago Musters) y los $400 \mathrm{~m}$ s.n.m. (100 m sobre el nivel del lago Musters) que forman una franja estrecha con un máximo de $5 \mathrm{~km}$. El fondo de valle es muy extenso, entre 20 y $50 \mathrm{~km}$ en un relieve plano entre las cotas de 280 hasta los $255 \mathrm{~m}$ s.n.m. Estos lagos están separados por la sierra Silva y distantes entre sí unos siete $\mathrm{km}$ en el Norte y $20 \mathrm{~km}$ en el extremo Sur. El lago Musters es relativamente profundo, con $40 \mathrm{~m}$ de profundidad máxima y $20 \mathrm{~m}$ de media, y una superficie de 440 $\mathrm{km}^{2}$. Por su parte el lago Colhué Huapi puede ser considerado como una laguna con una profundidad máxima de $5 \mathrm{~m}$ y una media de $2 \mathrm{~m}$, y su superficie es de $810 \mathrm{~km}^{2}$. Ambos son lagos productivos respectivamente mesotrófico y argilóroficos, en los que se practica la pesca tanto deportiva como comercial. Estos lagos son alimentados por el río Senguer, el lago Musters en forma directa y el lago Colhué Huapi indirectamente a partir de un brazo denominado Falso Senguer. A partir del último lago la cuenca continuaba por el río Chico, aunque desde 1930 y 1940 por el desecamiento del lago y la formación de médanos se interrumpió la comunicación con el río Chico lo que provocó la transformación en una cuenca endorreica (Coronato, 2003).

Según Césari y Simeoni (1994), los lagos se originan a partir de una depresión formada por procesos tectónicos y erosivos. Inicialmente el río Senguer corría más al sur por el Valle Hermoso, hasta que el río fue captado por erosión retrocedente y las aguas 


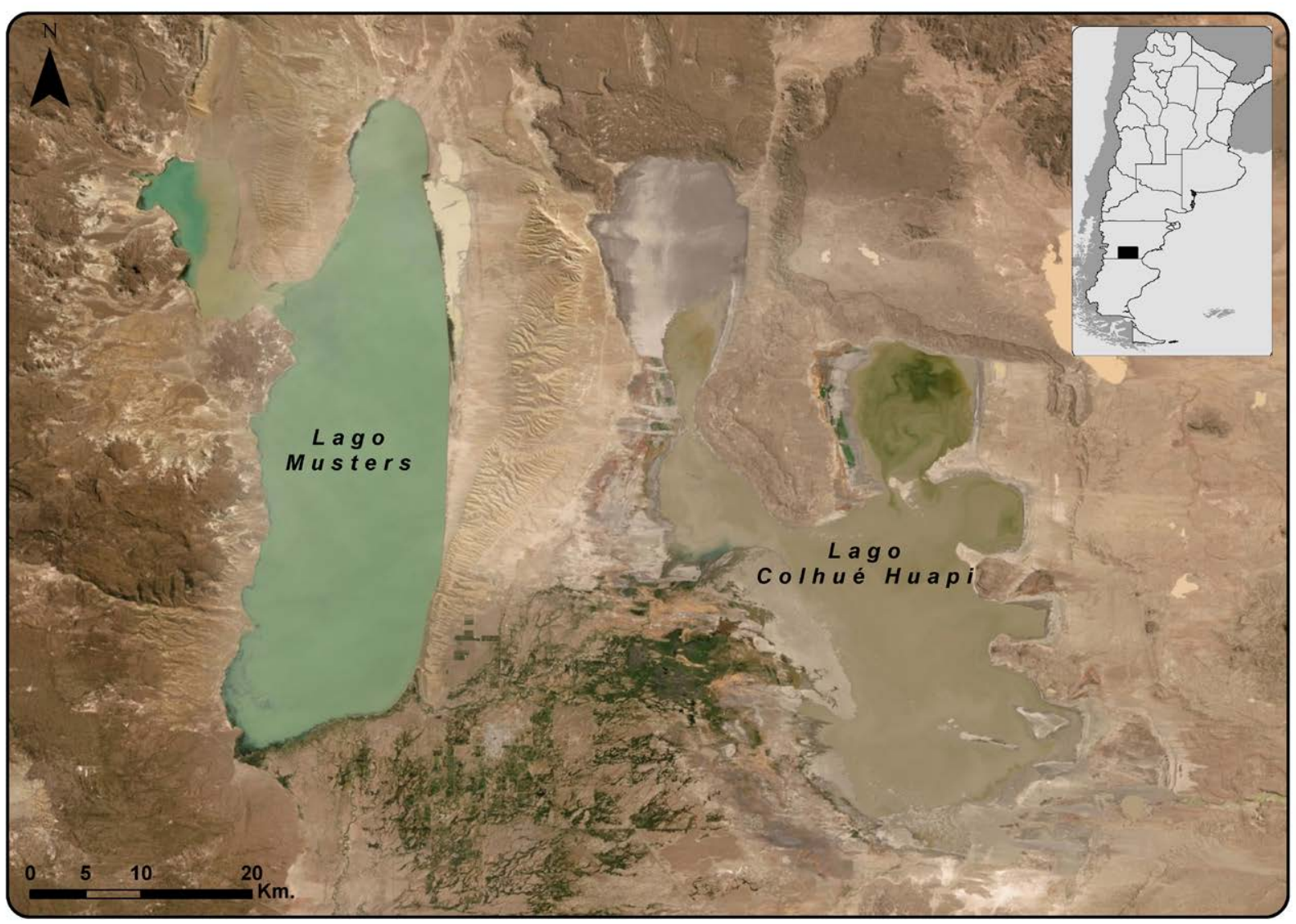

Figura 1. Ubicación geográfica del bajo de Sarmiento.

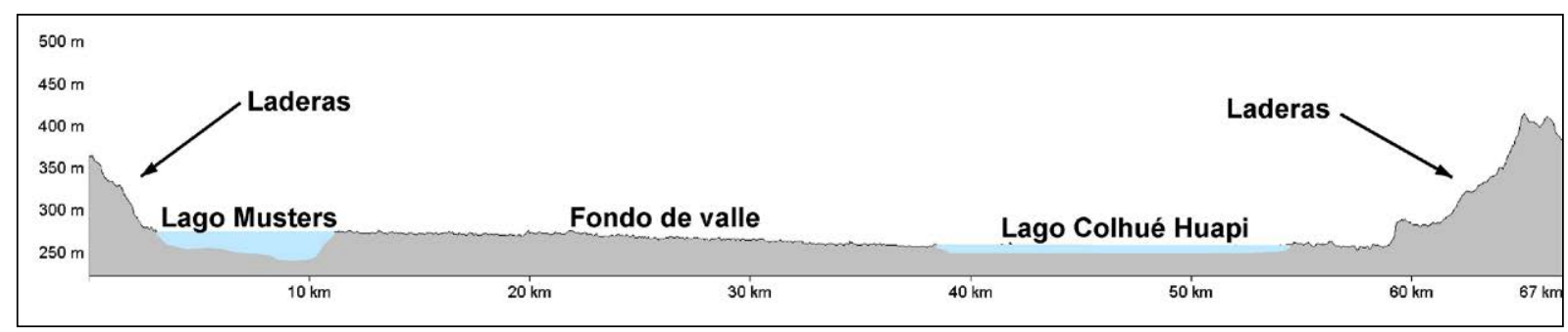

Figura 2. Perfil de elevación de un sector del bajo de Sarmiento.

ingresaron inundando el bajo a finales del Pleistoceno o inicios del Holoceno, lo que generó el paleolago Sarmiento, que habría alcanzado una altura de unos $60 \mathrm{~m}$ s.n.LM (metros sobre el nivel del lago Musters) (González Díaz y Di Tommaso, 2014; Montes, Rodríguez y Domínguez, 2017; Scordo et al., 2017) aunque descendió posteriormente y dejó paleoplayas a 30 y 10 m s.n.LM.

Frente al problema de ausencia de cronología para el paleolago y sus diferentes niveles, se intentó datar varias muestras de sedimentos de fondo de lago y dos vértebras probablemente de Lama guanicoe encontradas dentro de una paleoplaya, pero en todos los casos fue imposible por la escasez de materia orgánica. Esto obligó a recurrir a una metodología indirecta basada en dataciones de sitios arqueológicos, su distribución y relación con geoformas. A partir de ello, se construyó un modelo de evolución del 
paleolago (Moreno et al., 2016) que a continuación se resume complementándolo con algunos datos novedosos.

Para inferir la cronología de niveles más elevados del paleolago, se utilizó la información de los sitios PS 15 y PS 20 ubicados en el pedimento sur del bajo (Figura 3). PS 15 se halla dentro de un médano que apoya sobre un cordón litoral en la cota de $30 \mathrm{~m}$ s.n.LM. La orientación del médano es noreste- suroeste y no se adecua a la dirección del viento predominante del Oeste. Si se le suma que su posición se halla sobre el cordón litoral, es posible asegurar que se trata de un médano litoral, lo que indica que en el momento de su formación la costa del lago se hallaría próxima al PS 15 y por debajo de los 30 m s.n.LM. (la costa actualmente está a $15 \mathrm{~km}$ si tomamos la del lago Colhué Huapi y a $30 \mathrm{~km}$ la del lago Musters).

Este médano, que contiene al sitio PS 15, presenta un frente erosionado en donde se observa una capa de rodados aplanados de la paleoplaya, por encima arena consolidada, un nivel de tefra, sobre ella nuevamente arena consolidada, y por último un médano activo. Inicialmente se dató un nivel de fogón registrado en la arena consolidada por encima de la tefra dando una antigüedad de $3050 \pm 60$ AP (LP2720 , carbón). Es muy posible que esta tefra se corresponda con la hallada en PS 20 donde se dató un fogón en $4500 \pm 80$ AP (LP-2722, carbón) por debajo del nivel de cenizas volcánicas. De ser así, la base del médano litoral en el que se halla PS 15 debería tener una antigüedad similar y cerca de esta fecha debería estar el nivel del lago de $30 \mathrm{~m}$ s.n.LM. (Moreno et al., 2016). Esta inferencia fue confirmada por una nueva datación de PS 15 . Se trata de un fogón por debajo del nivel de tefra y a pocos centímetros de profundidad respecto a la paleoplaya. Se excavó una pequeña superficie de $50 \times 50 \mathrm{~cm}$ recuperándose carbón que fue fechado en $4560 \pm 150$ años AP (LP-3462). Por lo tanto, el nivel del lago cercano a los $30 \mathrm{~m}$ s.n.LM sería algo anterior a esta fecha. Por la cronología es posible que el nivel de tefra corresponda con la detectada en Alero Dasovich y atribuido a la erupción Hudson II (Aguerre, Andrieu y Lantanos, 2017).

Con respecto a los niveles más bajos, se analizaron los sitios arqueológicos según su posición topográfica (laderas o fondo de valle) y cronológica (anterior o posterior al 1500 AP), y se observó que los sitios arqueológicos anteriores a 1500 AP se sitúan en las laderas, en tanto que los posteriores a esta fecha, si bien se registraron algunos en las laderas, son más frecuentes en el fondo del valle (véase Moreno et al., 2016). El test de Fisher exacto indica que la distribución cronológica y espacial de los sitios no es azarosa. La explicación más probable es que cerca del 1500 AP el paleolago tenía un nivel de $10 \mathrm{~m}$ s.n.LM que cubría con sus aguas todo el fondo del valle e impedía la ocupación humana. Considerando la topografía del bajo, esta cota se corresponde con un cordón litoral más marcado y continuo (Moreno et al., 2016). Un nuevo fechado realizado en el sitio Barranca Martínez 1, con una antigüedad de $2360 \pm 80$ años AP (LP- 3323, carbón), da sustento a esta posibilidad. El sitio está ubicado al pie de un acantilado inactivo en cercanías del nacimiento del río Chico (Figura 3); se trata de una terraza adosada formada en su porción inferior por sedimentos de fondo de lago. Dentro de la terraza se halló un fogón, a $8 \mathrm{~m}$ s.n.LM y unos pocos artefactos líticos a partir de un sondeo de $50 \times 50 \mathrm{~cm}$. Por supuesto, la combustión ocurrió en un momento de descenso del lago; luego de ocurrido esto el lago volvió a subir y continuaron depositándose niveles de sedimento de fondo de lago. El hecho de no encontrarse ningún depósito de sedimentos terrestres indica que se trató de un fenómeno de poca duración, quizás estacional, lo que a su vez señala que el nivel normal del lago no debería estar muy por encima. Por otro lado, como se señaló más arriba, el Colhué Huapi es un lago sujeto a grandes fluctuaciones y además son muy frecuentes las ocupaciones en la planicie de inundación. Esta es la única datación directa del paleolago lograda hasta el momento. 


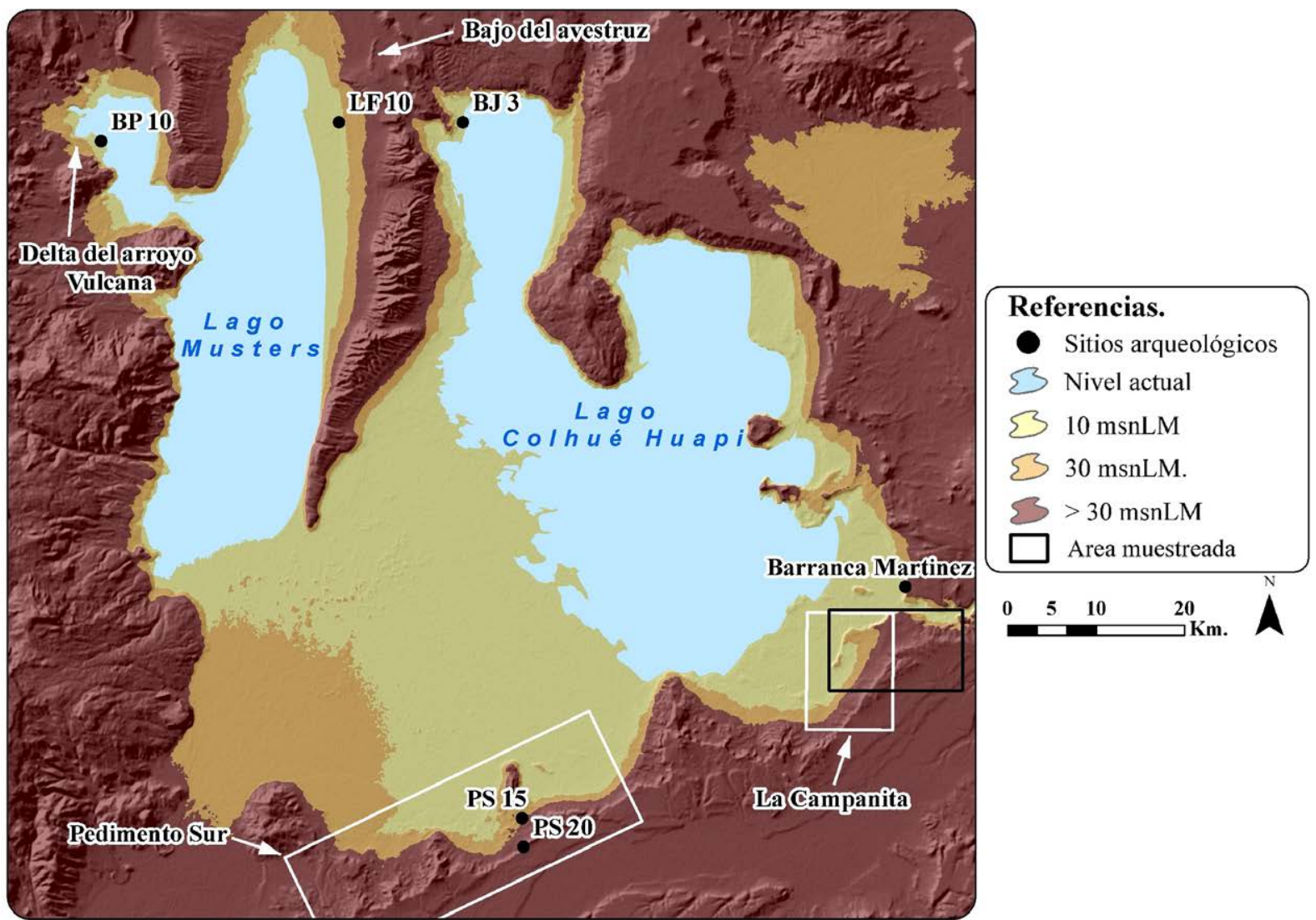

Figura 3. Reconstrucción de cotas del paleolago y ubicación de sitios arqueológicos.

Esta reconstrucción es coherente y complementaria de los estudios palinológicos realizados en el lago Colhué Huapi. En el sector del Boliche de Jerez (noroeste del lago), Trivi de Mandri y Burry (2007) señalaron un nivel del lago más elevado antes de $1586 \pm 40$ AP y un posterior descenso, incluso por debajo del nivel actual que se correspondería con la Anomalía Climática Medieval, y por último una nueva elevación posterior. Por su parte, Fabiani, Burry y Escalante (2014), trabajaron un perfil en el yacimiento La Campanita (sudeste del lago) con resultados similares, identificando un nivel más alto hacia el $1711 \pm 35$ AP. A partir de ca. 1200 AP detectaron una disminución del nivel con fluctuaciones, luego un descenso marcado hacia el $970 \mathrm{AP}$ con grandes fluctuaciones entre ese momento y ca. 195 AP, y finalmente una elevación hacia niveles históricos a partir de ese momento. Estas fluctuaciones tardías están avaladas por la detección de sitios arqueológicos en la planicie de inundación, que de acuerdo a los mapas cartográficos de la década de 1940 se hallaban sumergidos (Arrigoni, 2001). Asimismo, estudios basados en datos históricos e imágenes satelitales señalan un progresivo desecamiento del lago Colhué Huapi: hacia los años 2000-2001 el lago desapareció casi en su totalidad, luego registró una recuperación de su nivel en el año 2006, y volvió a descender en el 2011 manteniéndose bajo hasta la actualidad (Coronato, 2003; Llanos et al., 2016; Montes et al., 2017). Cabe mencionar que el vecino lago Musters no registra fluctuaciones drásticas, principalmente por la mayor profundidad y la conexión más directa con el río Senguer.

En resumen, el esquema de evolución propone que en el Holoceno medio (ca. 5000 AP) el nivel del paleolago se hallaba unos $30 \mathrm{~m}$ s.n.L.M, cerca del 2000 AP habría descendido hasta los $10 \mathrm{~m}$ s.n.L.M, y con posterioridad al 1600 AP se habrían formado los lagos con niveles similares a los actuales dejando expuesto gran parte del fondo del valle (Figura 3). Cerca del 1000 AP el lago Colhué Huapi, y quizás el lago Musters, 
habrían descendido por debajo de sus niveles actuales recuperándose luego hasta alcanzar el nivel de la década del 1940.

Respecto de la utilización de la cota como marco de referencia para evaluar la cronología de la actividad pesquera, cabe mencionar que no se asume que todos los sitios o artefactos hallados en las laderas sean antiguos, ya que éstas estuvieron disponibles para la ocupación humana por cerca de 5000 años. No obstante, de acuerdo a los nueve fechados radiocarbónicos obtenidos en las laderas, siete son anteriores al 1600 AP y sólo dos son posteriores (Moreno et al., 2016, tabla 1). Es base a esto se puede plantear la alta probabilidad de que una parte importante del registro de las laderas corresponda a ocupaciones del Holoceno medio o incluso más antiguas. Por su parte, de acuerdo al esquema de evolución del paleolago, se plantea que el registro arqueológico del fondo de valle presentará una antigüedad máxima de 1500 AP.

\section{Los pesos líticos como indicadores de pesca}

En el bajo de Sarmiento se han hallado diversos artefactos atribuibles a la pesca en poblaciones cazadoras-recolectoras. Los más notorios son los arpones unidentados fijos construidos con hueso de mamíferos terrestres, muy similares a los de la costa atlántica de Tierra del Fuego (Torres, 2007). Lamentablemente son pocas piezas, los hallazgos fueron hechos por particulares, y en muchos casos se carece de información exacta de procedencia más allá de indicaciones generales.

A diferencia de los arpones, los pesos líticos son muy frecuentes tanto en las colecciones como en el registro arqueológico relevado por nuestras investigaciones sistemáticas del área. A partir de una muestra relativamente reducida, Reyes y Svoboda (2016) identificaron cuatro tipos de pesos líticos en el bajo: el tipo A, globular o subglobular con surco o semisurco en el eje transversal sobre roca sedimentaria; el tipo B, globular o subglobular con surco o semisurco en el eje transversal sobre roca ígnea; el C de sección transversal plana y muescas en eje transversal, generalmente sobre rodados de playa, y el D globular con surco subecuatorial (Reyes y Svoboda, 2016, Figura 4). Preliminarmente y considerando su peso, morfología y condiciones de hallazgo, los del tipo $\mathrm{C}$ fueron interpretados como pesos de red y los demás tipos tanto podrían ser pesos de líneas como formar parte de redes más pequeñas (Reyes y Svoboda, 2016).

Posteriores trabajos de campo permitieron ampliar enormemente esta muestra inicial. Además de los cuatro tipos morfológicos definidos se agrega el tipo $\mathrm{E}$, caracterizado por una sección transversal plana, con muescas en el eje transversal y en el sector medio, sobre bloques chatos previamente formatizados, muy similares en tamaño, forma y peso al grupo $\mathrm{C}$, de los que diferencian por la forma base y materia prima (Reyes, Peralta González y Svoboda, 2016). Se pudo constatar que los del tipo C y E (Figura 4) son muchos más numerosos que los restantes, aun considerando que los provistos de surco (A, B y D) pueden ser más atractivos para los coleccionistas. En muchos casos se los halló agrupados en conjuntos de hasta 16 artefactos (Figura 4B) y con frecuencia están alineados, reforzando la interpretación de pesos de red (Svoboda, 2017). Los pocos ejemplares de los otros tipos normalmente aparecen aislados.

A partir de las siguientes propiedades se considera a los pesos líticos como indicadores confiables de la actividad pesquera porque:

1. Son durables, ya que están construidos en rocas relativamente duras a diferencia de los arpones de hueso mucho menos durables particularmente en condiciones de exposición subaéreas. 
2. Son poco atractivos para los coleccionistas, ya que presentan escasas modificaciones; esto es particularmente cierto para el tipo $\mathrm{C}$.

3. Requieren una baja inversión de trabajo, por lo que se descartarían en el lugar de uso.

4. Por su tamaño y su peso (con una media próxima a los 400 g; Reyes y Svoboda, 2016, figura 4) es poco probable que fueran trasladados intencionalmente por pescadores prehistóricos.

5. Tienen baja posibilidad de ser trasladados para reutilización como artefactos o como fuente de materia prima. Respecto a lo primero no se registraron casos de reutilización sobre estos artefactos. En cuanto a su reutilización como materia prima, generalmente están fabricados sobre rodados de mala calidad para la talla y amplia distribución espacial, o en rocas sedimentarias también frecuentes y de nula calidad para la talla.

6. Finalmente, varios autores los vinculan a diferentes sistemas de pesca (Cardillo, Scartascini y Zangrando, 2015; Gómez Otero, 2006; Mansur, 2007; Massone y Torres, 2004; Scartascini y Cardillo, 2009; Torres, 2007).

A
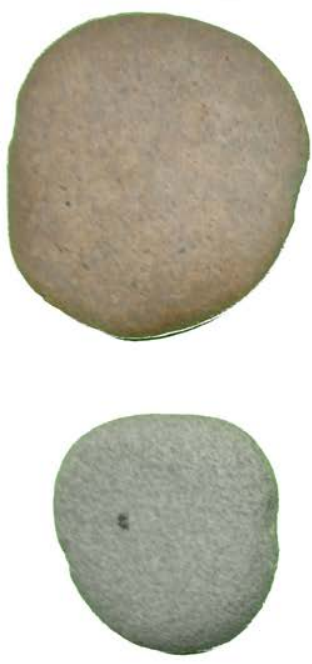
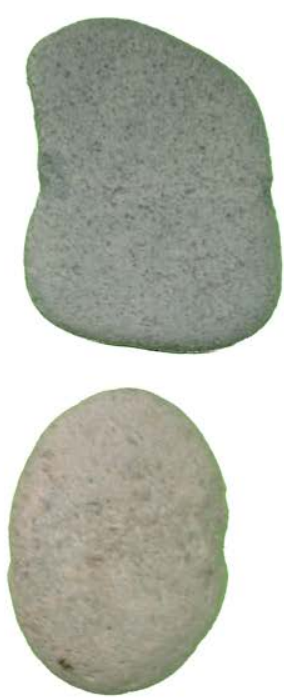

B
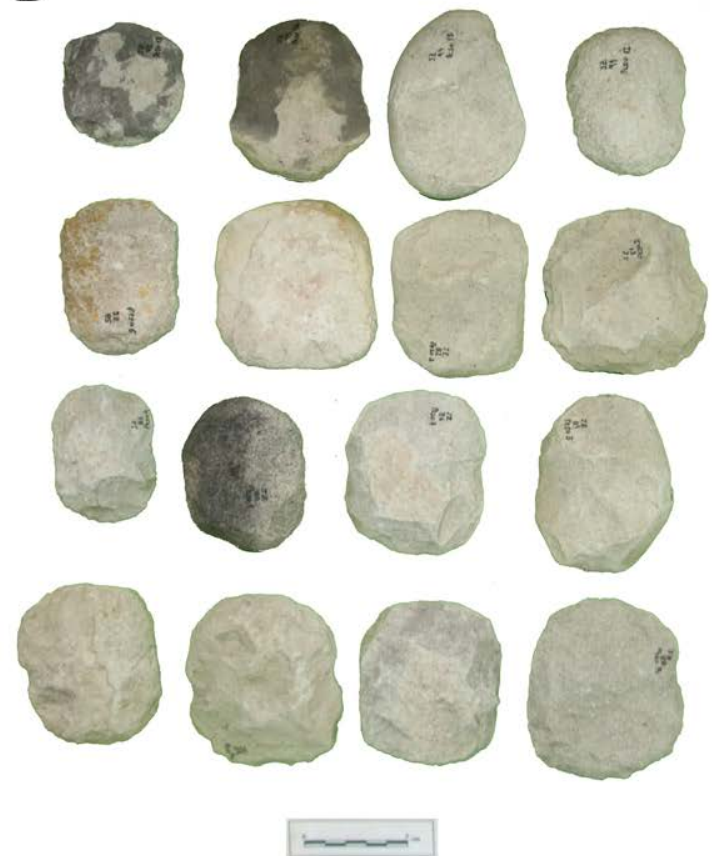

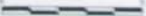

Figura 4. Pesos líticos más frecuentes del registro de superficie del lago Colhué Huapi. A) pesos líticos confeccionados sobre canto rodado (tipo C); b) y sobre forma base formatizada (tipo E).

\section{Metodología}

A fin de poner a prueba la presunción de la pesca como un importante componente de la subsistencia de cazadores-recolectores hacia el Holoceno tardío final, se realizaron investigaciones en un sector de la estancia La Campanita, al sur del nacimiento del río Chico (Figura 3). El área fue seleccionada por la poca pendiente del terreno y la 
presencia de varios sectores con alta visibilidad arqueológica. Es un sector en el cual las laderas descienden gradualmente desde el borde del Valle Hermoso, a unos $80 \mathrm{~m}$ s.n.LM, hasta la planicie de inundación del lago. Este descenso está interrumpido por un acantilado inactivo a $10 \mathrm{~m}$ s.n.LM que separa las laderas del fondo del valle. Las laderas están formadas por sedimentos que alternan médanos antiguos, paleosuelos y antiguos cordones litorales, todo cubierto por un depósito arenoso en forma de manto más o menos uniforme. El fondo del valle está cubierto por un suelo hidromórfico sobre el que, en sectores puntuales, se depositan dunas modernas.

El sector se dividió en subsectores llamados La Campanita Alto (LCA) (más de $30 \mathrm{~m}$ s.n.LM), Medio (LCM) (entre 30 y 10 m s.n.LM) y Bajo (LCB) (menos de 10 m s.n.LM), asignables de acuerdo al modelo de evolución del lago al Holoceno medio (antes de 5000 AP), Holoceno medio final y tardío inicial (5000 a 2000 AP) y Holoceno tardío final (luego de $1500 \mathrm{AP}$ ), respectivamente.

El diseño de muestreo contempló una estrategia azarosa (transecta) y otra orientada (localización de hoyadas de deflación y otros rasgos erosivos). En este sentido, por un lado se realizó una transecta ( $\mathrm{T} 1$ ) de $13 \mathrm{~km}$ de longitud en dirección Este-Oeste siguiendo la pendiente del terreno cuyo trazado atraviesa la misma distancia en cada subsector (4 km cada uno) (Figura 5). Estuvo a cargo de cuatro personas que caminaron separadas por $20 \mathrm{~m}$ entre sí cubriendo una superficie de $80 \mathrm{~m}$ de ancho. El total de la superficie relevada es de 104 hectáreas, de las cuales 36 corresponden a cada subsector. Sobre el final de la transecta, en el subsector Bajo, se debió sortear una laguna temporaria generando una curva (Figura 5). Las variables consignadas fueron el tipo de geoforma, tipos de sedimentos y visibilidad superficial estimada a partir de las coberturas vegetal y de médanos modernos.

Dado que durante el relevamiento a lo largo de la T1 se observaron diferencias en la cobertura entre los subsectores (ver abajo), se planteó la realización de prospecciones orientadas en los rasgos erosivos, principalmente en médanos deflacionados. El primer paso fue la detección de estos sectores en el paisaje mediante imágenes satelitales y,

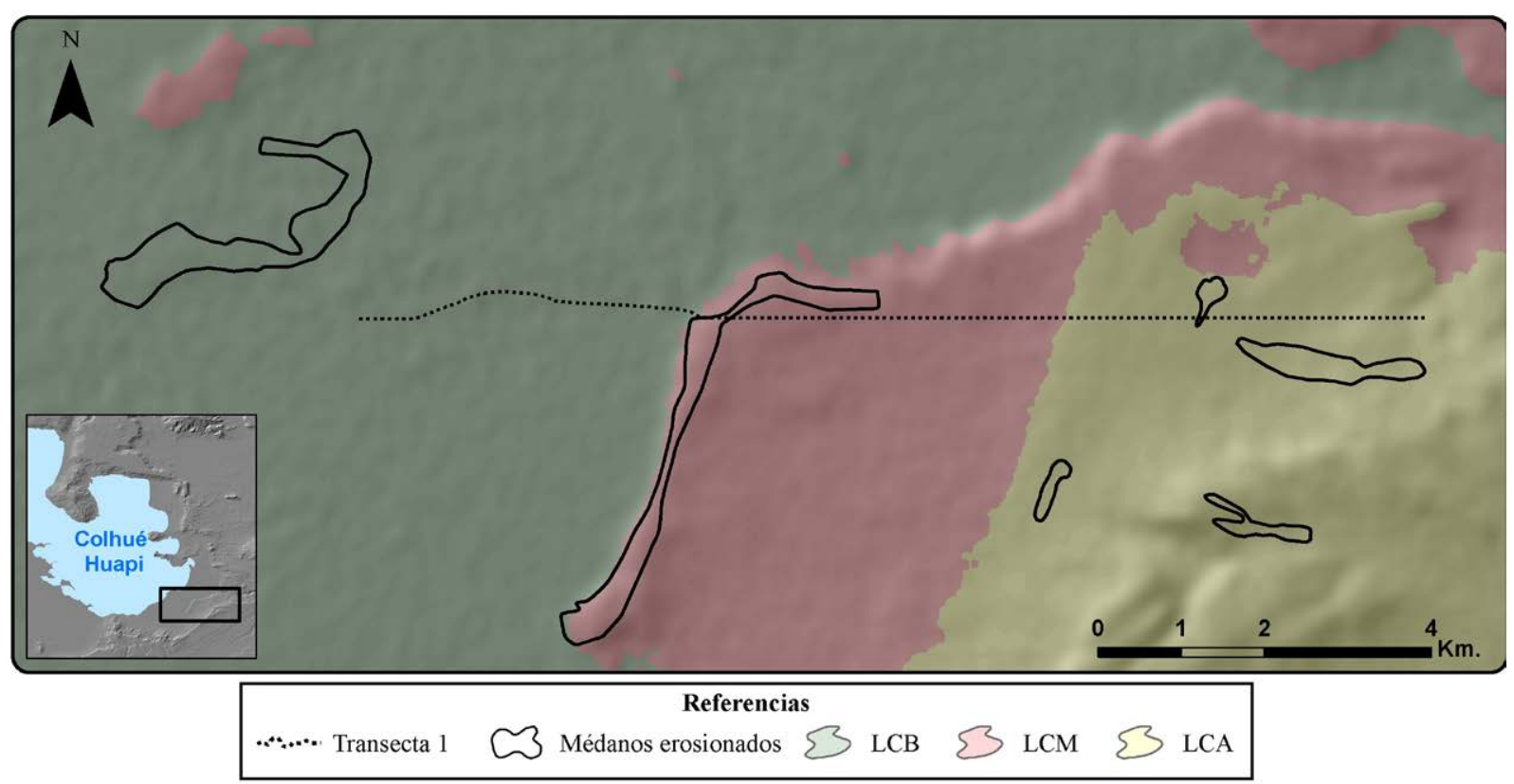

Figura 5. Transecta 1 (T1) y localización de los subsectores de La Campanita. LCB= La Campanita Alto (más de $30 \mathrm{~m}$ s.n.LM); LCM = La Campanita Medio (entre 30 y 10 m s.n.LM); LCB= La Campanita Bajo (menos de $10 \mathrm{~m}$ s.n.LM). 
posteriormente, su prospección. Este trabajo fue realizado por cinco personas durante 15 días de campo.

En cuanto al registro de datos de los pesos líticos y sitios arqueológicos, tanto en la transecta $T 1$ como en los médanos erosionados se utilizaron planillas estandarizadas. Respecto de los pesos líticos se consignó la posición, materia prima, tipo y condición (agrupado o aislado). En el caso de los sitios se registró posición, tipo de sedimento, tamaño y densidad aproximado estratigrafía, fogones, cerámica, materias primas, entre otros. También se registraron los problemas de visibilidad y potenciales alteraciones postdepositacionales. Por otro lado, en los sitios donde fue posible se tomaron muestras de sedimentos de fogones mediante sondeos de $50 \times 50 \mathrm{~cm}$, a fin de realizar estudios faunísticos y obtener dataciones radiocarbónicas. Estos sedimentos fueron tamizados en laboratorio con zaranda de agua de $2 \mathrm{~mm}$ para extraer carbones y asegurarse de no perder ningún resto considerando el pequeño tamaño de los huesos de peces.

Finalmente, para ampliar la muestra, se sistematizó la totalidad de la información de las prospecciones realizadas desde el inicio del proyecto en el año 2005. Para ello se tomó la precaución de que estos datos presentaron cierta dificultad de estandarizar debido a los cambios en la metodología relacionados con los distintos objetivos de cada fase del proyecto y a la acumulación de experiencia. Sin embargo, y considerando que la búsqueda de pesos líticos estuvo en agenda desde el inicio del proyecto porque se los había observado en colecciones (Moreno, Videla, Pérez Ruiz, Asencio y Leonforti, 2007), es que se pudo hacer uso de esta información. Fundamentalmente, se tomó tal información agrupándola en laderas y fondo de valle. Las laderas incluyen las cotas equivalentes a LCA y LCM y el fondo de valle a LCB. Se tuvo en cuenta la intensidad de la investigación en cada sector estimada a partir de la cantidad de campañas realizadas.

\section{Resultados}

Como primer resultado se señala que la mayoría de los pesos hallados en la $\mathrm{T} 1$, los médanos erosionados y trabajos previos, corresponden al tipo $\mathrm{C}$ y $\mathrm{E}$. La prospección sobre la T1 permitió registrar un sitio en LCA, uno en LCM y cuatro en LCB. Los pesos líticos suman 52 , todos provenientes de LCB (Tabla 1 ). Si bien el resultado parece ser contundente, es necesario tener en cuenta que en los subsectores LCA y LCM la visibilidad es menor y sólo se registraron sitios en los pocos lugares que no presentaban cobertura de médanos modernos. En el subsector LCB los sedimentos son, en general, suelos hidromórficos con menor cobertura de médanos modernos, lo que supone a priori mayor visibilidad.

\begin{tabular}{|l|c|c|c|c|c|c|c|}
\hline & Sitios & Pesos líticos & $\begin{array}{c}\text { Peso lítico / } \\
\text { Sitio }\end{array}$ & $\begin{array}{c}\text { Pesos líticos } \\
\text { (\%) }\end{array}$ & $\begin{array}{c}\text { Superficie } \\
\text { (hectárea). }\end{array}$ & $\begin{array}{c}\text { Densidad sitio } \\
\text { (Sitio/hect.) }\end{array}$ & $\begin{array}{c}\text { Densidad de } \\
\text { Pesos líticos } \\
\text { (PL/hect.) }\end{array}$ \\
\hline LCA & 1 & 0 & 0 & 0 & 36 & 0,02 & - \\
LCM & 1 & 0 & 0 & 0 & 36 & 0,02 & - \\
LCB & 4 & 52 & 13 & 100 & 36 & 0,11 & 1,4 \\
\hline
\end{tabular}

Tabla 1. Resultados de hallazgos arqueológicos de la Transecta 1 (T1) en el lago Colhué Huapi.

Las prospecciones en los médanos erosionados comprendieron 221 hectáreas en total, de las cuales 50 corresponden a LCA; 74 a LCM y 87 a LCB (Tabla 2). Se registraron 15 sitios en LCA, 11 en LCM y 10 LCB, en este último sector se hallaron 46 pesos líticos, uno en LCM y ninguno en LCA. La cantidad de sitios por hectárea dio 0,3 para LCA y 
0,1 para la LCM y LCB. La cantidad de pesos líticos por hectárea prospectada fue de 0 para LCA; 0,01 para LCM y 0,53 para LCB (Tabla 2 ).

\begin{tabular}{|l|c|c|c|c|c|c|c|c|c|}
\hline & Sitios & Pesos & Peso/sitio & Peso \% & Hect. & Sitio/Hect. & Peso/Hect. & Fogones & $\begin{array}{c}\text { Fogones } \\
\text { con peces }\end{array}$ \\
\hline LCA & 15 & 0 & 0 & 0 & 50 & 0,3 & 0 & 3 & 0 \\
LCM & 11 & 1 & 0,1 & 2 & 74 & 0,1 & 0,01 & 1 & 0 \\
LCB & 10 & 46 & 4,6 & 98 & 87 & 0,1 & 0,53 & 3 & 2 \\
\hline
\end{tabular}

Tabla 2. Resultados de las prospecciones en médanos erosionados en el lago Colhué Huapi.

Los resultados de la Tabla 2 permiten descartar la posibilidad de que la cantidad de sitios y pesos líticos registrados se expliquen a partir de problemas de visibilidad ya que se trató de geoformas similares y afectadas por los mismos procesos, no a diferentes superficies prospectadas y además en LCA y LCM se hallaron más sitios que en LCB.

Respecto del impacto de los coleccionistas, éste es claramente mayor en LCB según lo expresado por los propietarios de los campos. La explicación de este fenómeno es la notable red de caminos vinculados a la actividad petrolera, que facilita el acceso a los sitios de mayor tamaño, densidad y riqueza artefactual. Los otros dos sectores son de difícil acceso y los sitios son pequeños y poco densos. Es decir que sería más esperable que faltaran pesos líticos (y artefactos en general) en la LCB que en LCM y LCA.

Se obtuvieron siete muestras de fogones (tres en LCA en médanos erosionados, tres en LCB en sedimento hidromórfico, y uno en LCM también en un médano erosionado) cuyos resultados preliminares indicaron que sólo dos sitios contienen restos de peces y están en LCB. La única datación disponible es del sitio LCM 1 y arrojó una antigüedad de $4080 \pm 90$ años AP (LP- 3471, carbón), lo que es coherente con la asignación temporal propuesta para estos sitios, según el modelo del paleolago. La presencia de cerámica en el $40 \%$ de los sitios de LCB y su ausencia total en los otros dos sectores también avala esta ubicación cronológica, considerando que, como ya se señaló, LCB es el sector más impactado por los coleccionistas.

En las prospecciones previas en el perilago del lago Musters (Tabla 3 ) en donde se trabajó durante siete años realizando nueve campañas, se registraron 103 sitios y cuatro pesos líticos (Moreno et al., 2007). En el Colhué Huapi las prospecciones se vienen realizando desde hace dos años en un total de dos campañas, en las que fue posible registrar 24 sitios y 265 pesos líticos. Cabe aclarar que se cuentan para este caso las campañas hasta el año 2015 , ya que a partir de ese momento se dejaron de registrar estos artefactos, salvo en ocasiones especiales como los trabajos en La Campanita, ya desarrollados. En las laderas de la localidad de Bajo del Avestruz se trabajó durante dos años y se realizaron dos campañas, cuyos resultados son 15 sitios y ningún peso. En Pedimento Sur, que incluye también el Bosque Petrificado Sarmiento y la zona del cerro Purichelli, se trabajó tres años con cinco campañas y fueron detectados 96 sitios y cuatro pesos líticos.

\begin{tabular}{|l|c|c|c|c|c|}
\hline & Campañas & Sitios & Pesos & Pesos/sitio & $\%$ Pesos \\
\hline Laderas (LCA +LCM) & 7 & 111 & 4 & 0,04 & 1,4 \\
Fondo de valle (LCB) & 9 & 127 & 269 & 2,12 & 98,5 \\
\hline
\end{tabular}

Tabla 3. Resultados de prospecciones previas en los lagos Colhué Huapi y Musters. 
Si agrupamos estos datos vemos que la inmensa mayoría -el $98 \%$ - de los pesos líticos están en el fondo de valle, en tanto que en las laderas son muy escasos. Esto no guarda relación con la cantidad de trabajo realizado, similar en ambas unidades de paisaje, ni con la visibilidad, como lo demuestra el número de sitios registrados. Sin embargo, si consideramos separadamente el perilago del Colhué Huapi vemos que de allí provienen el $97 \%$ de los pesos, en tanto que en el lago Musters sólo presenta el $1,4 \%$, cifra similar a la de las laderas.

\section{Discusión}

Según los resultados obtenidos en la $\mathrm{T} 1$, las prospecciones en los médanos erosionados y las prospecciones previas, el $100 \%$, el $98 \%$ y el $98,5 \%$ respectivamente de los pesos líticos provienen del fondo del valle. El hallazgo de estos artefactos asociados a los sistemas de pesca no está afectado por las diferencias en la visibilidad, el accionar de coleccionistas, ni la diferente intensidad de los trabajos arqueológicos. A esto se suma que sólo en los fogones de LCB ( $y$ en general en los sitios del fondo del valle) se hallaron restos de peces, aunque es necesario considerar los problemas de conservación de los sitios en las laderas. Algunos de estos sitios (Valle Hermoso 4 y Boliche de Jerez 3) se estudiaron, y los análisis zooarqueológicos arrojaron una abrumadora cantidad de restos de peces con evidencias de aprovechamiento antrópico (Svoboda, 2019; Svoboda y Moreno, 2018).

La presencia restringida de los pesos líticos en el fondo de valle genera dos nuevos problemas: por un lado, el significado de los pocos pesos de red en las laderas y, por otro, la diferencia en la frecuencia de pesos entre los perilagos del Musters y Colhué Huapi. En cuanto al primer problema, puede pensarse que en la aproximación inicial (Reyes y Svoboda, 2016) se contaba con muy poca información ya que los trabajos en el lago Colhué Huapi -donde se encontraron la inmensa mayoría de los pesos- no habían empezado. En la actualidad, con una muestra mucho más grande, estos pocos pesos pierden significado. Por otro lado, y considerando la escasa pendiente del fondo del valle, no puede descartarse que en algún momento del Holoceno tardío final haya ocurrido una inundación excepcional que habría convertido el fondo del valle en un humedal donde se pudiera pescar. Es de destacar que al menos cuatro de los cinco artefactos del Pedimento Sur, aunque se hallen por encima de los $10 \mathrm{~m}$ s.n.LM están horizontalmente muy próximos al fondo del valle. Finalmente, no puede descartarse que se trate de las primeras etapas, experimentales y poco frecuentes, de la pesca. Lamentablemente, los sitios a los que se asocian estos pesos líticos son superficiales, lo que no permite datarlos ni obtener muestras arqueofaunísticas.

Más complejo de explicar es el problema de las diferencias de frecuencia de pesos entre los perilagos. En efecto, en el Musters se trabajó más tiempo, hay gran cantidad de sitios registrados y sin embargo los pesos de red son muy escasos. Asimismo, debemos considerar que pueden existir diferencias en la visibilidad, ya que gran parte de la costa oeste del lago Musters está cubierto de médanos vegetados mientras que el sector sur presenta una notable formación de suelo poco erosionado. En tanto, en la costa este y norte la visibilidad es similar a la del lago Colhué Huapi con suelos y médanos erosionados (Moreno et al., 2007). Por otro lado, es muy interesante incluir en esta discusión el caso de la localidad de Delta del Arroyo Vulcana, intensamente trabajado entre los años 2008 al 2011, incluyendo prospecciones, sondeos y excavaciones extensas (Moreno y Pérez Ruiz, 2010; Moreno y Svoboda, 2013; Peralta González, Moreno y Pérez Ruiz, 2014; entre otros). Esta localidad, ubicada en la costa noroeste del lago Musters, se incluye en un fan delta actualmente inactivo. El delta está en pleno proceso erosivo, evidenciado por la abundancia de plantas en pedestal y con las raíces expuestas, que deja grandes cantidades de materiales arqueológicos en superficie o en 
posición subsuperficial. Dentro de la localidad se realizó una excavación extensa del sitio DV1, datado ca. $1400 \mathrm{AP}$, el cual presenta registro de peces en bajas cantidades en relación a los guanacos. Pese a esto, es notable que en la localidad sólo se haya encontrado un peso lítico que, en rigor, fue hallado en las afueras de la localidad sobre la costa del lago. Por otro lado, corresponde a un peso del tipo D (Reyes y Svoboda, 2016), es decir, subglobular con surco completo subecuatorial. Algo similar ocurre con la cerámica: a diferencia de la gran cantidad de material lítico y óseo recuperado, solamente se hallaron dos tiestos. Esta escasez de cerámica -cuya datación más antigua en Patagonia central remonta a 1200 AP (Schuster, 2011)- sumada a las dataciones disponibles, sugiere que la mayor parte del registro de esta localidad sería anterior a esta fecha. Esto se contrapone al componente más moderno de Laguna de la Flecha 10 (costa noreste del Musters), posterior al 1000 AP, en la cual, aunque se presenta una densidad mucho menor de artefactos, se hallaron dos pesos líticos (Moreno et al., 2007).

A partir de esta información, puede plantearse una explicación que combina cronología, visibilidad y diferencias hídricas entre los lagos. Si la utilización de peces se hubiera iniciado en momentos muy tardíos (ca. 1000 AP), en el lago Musters sería poco visible ya que los sitios de esa cronología e inmediatos al lago estarían actualmente debajo del agua debido al descenso vinculado a la Anomalía Climática Medieval (ACM) y su posterior ascenso. En el caso del lago Colhué Huapi, los sitios próximos al lago y de esta antigüedad habrían quedado expuestos por la mayor sensibilidad a las fluctuaciones menores de este cuerpo de agua (Moreno et al., 2016).

Los datos obtenidos en el sitio Bahía Pastel 10, ubicado en el presente en la playa que apoya en un nivel arcilloso ubicado estratigráficamente por debajo de la berma de tormenta actual, permitieron corroborar un nivel más bajo del lago Musters. En este sitio se dató un fogón en 320 años AP. Sobre la berma mencionada es común encontrar artefactos líticos con poco rodamiento y en la costa inmediata -incluso dentro del agua- también restos óseos con marcado perimetral y artefactos líticos. Esta hipótesis permitiría también explicar la escasez generalizada de cerámica en el lago Musters en contraposición con su abundancia en el vecino Colhué Huapi.

\section{Conclusiones}

Como conclusión puede señalarse entonces que en el bajo de Sarmiento el aprovechamiento de peces lacustres a partir del empleo de redes es una estrategia económica asignable al Holoceno tardío final. Si tomamos en cuenta que en los numerosos relatos de viajeros de los siglos XVIII y XIX no existen menciones a actividades de pesca (Moreno et al., 2007; Svoboda y Moreno, 2018) podemos acotar el fenómeno entre los 1500 y 250 años AP, sin que necesariamente abarque la totalidad de este segmento temporal. Para esto último debe tomar en consideración que el lago Colhué Huapi fue afectado por una gran sequía vinculada a la ACM (Fabiani et al., 2014; Trivi de Mandri y Burry, 2007); es decir que la pesca debería ser o inmediatamente anterior (ca.1500-1000) o posterior (900-250 años AP) o bien de ambos momentos. La evidencia de la localidad Delta del Arroyo Vulcana sugiere que cerca del 1400 AP hubo actividad de pesca, pero no demasiado importante y sin desarrollo de tecnología especifica.

Esta información permite organizar una secuencia provisoria. Entre el 5000 y el 1500 AP el paleolago cubre todo el fondo del valle y las ocupaciones, concentradas en las laderas, no presentan evidencia de pesca; probablemente serían cazadores de animales terrestres, y según la evidencia de otros sectores de Patagonia, básicamente de guanaco (Mengoni Goñalons, 1999; entre otros). Hacia el 1500 AP se desarticula 
el paleolago y se originan los dos lagos actuales; según el modelo de evolución del paleolago, se ocuparía el fondo del valle e iniciaría la experimentación de pesca sin desarrollo de tecnología específica evidenciado en la localidad Delta del Arroyo Vulcana; este proceso se interrumpiría con la ACM por desaparición del lago Colhué Huapi, aunque quizás haya continuado en el lago Musters. Luego de este fenómeno habría una nueva elevación del nivel de los lagos; en este momento la pesca adquiere importancia y se desarrollan tecnologías específicas. Hacia el 250 AP (siglo XVIII) los cazadores adoptan el caballo, lo que produjo el abandono de la pesca y una nueva concentración sobre recursos terrestres (Svoboda y Moreno, 2018).

Este esquema permite generar nuevas líneas de trabajo entre la que se destacan: a) afinar la cronología de los sitios del fondo del valle especialmente para verificar la interrupción vinculada a la ACM, b) continuar con la búsqueda en las laderas a fin de detectar sitios con buena conservación que permitan obtener muestras faunísticas confiables, c) iniciar la búsqueda de sitios sumergidos en el lago Musters.

\section{Agradecimientos}

A la Universidad Nacional de la Patagonia San Juan Bosco (UNPSJB), al Consejo Nacional de Investigaciones Científicas y Técnicas (CONICET) y a la Agencia Nacional de Promoción Científica y Tecnológica (ANPCyT), quienes financiaron estas investigaciones. Al Sr. Omar Oporto, al Sr. Héctor Martínez y al Sr. Chiche Martínez por recibirnos en sus establecimientos siempre de la forma más hospitalaria. A los y las integrantes del equipo de investigación que fueron parte de los trabajos de campo, a la Dra. Florencia Del Castillo Bernal por su asesoramiento y a los evaluadores por sus acertadas sugerencias. 


\section{Referencias citadas}

» Aguerre, A. M, J., Andrieu, M. y lantanos, N. (2017). Arqueología en Río Mayo, sudoeste del Chubut. Excavación en el Alero Dásovich: resultados preliminares. Intersecciones en Antropología, 18, 55-65.

» Arrigoni, G. I. (2001). Relevamiento arqueológico en el área de perilago del Colhué Huapi. (Evaluación de impacto arqueológica). Informe de impacto para GM \& Asoc. Manuscrito inédito.

»Cardillo, M., Scartascini, F. L. y Zangrando, A. F. (2015). Combining morphological and metric variations in the study of design and functionality in stone weights. A comparative approach from continental and insular Patagonia, Argentina. Journal of Archaeological Science: Reports, 4, 578-587. https://doi.org/10.1016/j.jasrep.2015.10.030

»Césari, O. y Simeoni, A. (1994). Planicies fluvioglaciares terrazadas y bajos eólicos de la Patagonia Central, Argentina. $13^{\circ}$ Symposium Latin-American Geosciences, Zentralblatt fur Geologie und Palaontologie, 1, 155-164.

»Coronato, F. (2003). El problema de la desecación del lago Colhué Huapi desde la Geografía Histórica. Trabajo presentado en Congreso Nacional de Geografía, 64 Semana de Geografía, Bahía Blanca, Argentina.

» Fabiani, A. C., Burry, L. S. y Escalante, A. H. (2014). Análisis de polen y microalgas en sedimentos de la márgen sur del lago Colhué Huapi, Chubut, Argentina. Una comparación entre orillas. Anales Instituto de la Patagonia, 42(1), 35-51.

» Gómez Otero, J. (2006). Dieta, uso del espacio y evolución en poblaciones cazadorasrecolectoras de la costa centro-septentrional de Patagonia durante el Holoceno medio y tardío. (Tesis Doctoral inédita), Universidad de Buenos Aires, Argentina.

»González Díaz, E. F. y Di Tommaso, I. (2014). Paleogeoformas lacustres en los lagos Musters y Colhué Huapí, su relación genética con un paleolago Sarmiento previo, centro sur del Chubut. Revista de la Asociación geológica Argentina, 71(3), 416-426.

» Llanos, M. E., Behr, S. J., González, J. H., Colombani, E. N., Buono, G. G. y Escobar, J. M. (2016). Informe de las variaciones del Lago Colhué Huapi mediante sensores remotos y su relación con las precipitaciones, http://inta.gob.ar/documentos/informe-de-las-variaciones-del-lagocolhue-huapi-mediante-sensores-remotos-y-su-relacion-con-las-precipitaciones (Acceso: 10 de diciembre, 2019).

» Mansur, E. (2007). Confección y uso de artefactos discoides en contextos de cazadoresrecolectores de Patagonia meridional: pesas para redes en el sitio HST01AM (Prov. de Santa Cruz, Argentina). En F. Morello, M. Martinic, A. Prieto y G. Bahamonde (Eds.), Arqueología de Fuego-Patagonia. Levantando piedras, desenterrando huesos... y develando arcanos (pp. 701708). Punta Arenas: Ediciones CEQUA.

» Massone, M. y Torres, J. (2004). Pesas, peces y restos de cetáceos en el campamento de Punta Catalina 3 (2.300 años AP.). Magallania, 32, 143-161. http://www.magallania.cl/index. $\mathrm{php} / \mathrm{magallania/article/view/1205}$

» Mengoni Goñalons, G. (1999). Cazadores de guanacos en la estepa patagónica. Buenos Aires: Sociedad Argentina de Antropología.

» Montes A., Rodríguez, S. S. y Domínguez, C. E. (2017). Geomorphology context and characterization of dunefields developed by the southern westerlies at drying Colhué Huapi shallow lake, Patagonia Argentina. Aeolian Research, 28, 58-70. https://doi.org/10.1016/j. aeolia.2017.08.001

» Moreno, E. J y Pérez Ruiz, H. (2010). Evidencias de utilización prehispánica de recursos fluviales en la cuenca del lago Musters (Chubut, Argentina). Trabajo presentado en XVII Congreso Nacional de Arqueología Argentina. Mendoza, Argentina 
» Moreno, E. J. y Svoboda, A. (2013). Explotación de peces y guanacos en el interior de Patagonia central: aportes del sitio Delta del Arroyo Vulcana 1 (lago Musters, Chubut). Cazadores Recolectores del Cono Sur. Revista de Arqueología, 7, 49-68.

» Moreno, E. J., Pérez Ruiz, H. y Ramírez Rozzi, F. (2016). Esquema cronológico y evolución del paisaje en el bajo de Sarmiento (Chubut). En F. Mena (Ed.), De mar a mar (pp. 477-485). Santiago de Chile: Ediciones CIEP/Ñire Negro.

» Moreno, E. J., Pérez Ruiz, H., Ramírez Rozzi, F., Reyes, M., Svoboda, A., Peralta González, S. y Herrera Santana, M. (2015). Primeros resultados de los trabajos arqueológicos en el lago Colhué Huapi (Chubut). Cuadernos del Instituto Nacional de Antropología y Pensamiento Latinoamericano, 24(2), 133-137. https://revistas.inapl.gob.ar/index.php/cuadernos/article/ view/635

» Moreno, E. J., Videla, B., Pérez Ruiz, H., Asencio, L. y Leonforti, V. (2007). Búsqueda de indicadores de diversificación económica prehistórica en la Cuenca del Lago Musters (Chubut, Argentina). Primeros resultados. En F. Morello, M. Martinic, A. Prieto y G. Bahmonde (Eds.), Arqueología de Fuego-Patagonia. Levantando piedras, desenterrando huesos... y develando arcanos (pp. 23-32). Punta Arenas: Ediciones CEQUA.

» Peralta González, S., Moreno, E. J. y Pérez Ruiz, H. (2014). La casa está en orden. Análisis espacial intrasitio de Delta Vulcana 1 (lago Musters, Chubut, Argentina) y movilidad de cazadores-recolectores en Patagonia central durante el Holoceno tardío. Magallania, 2(42), 141-153. http://www.magallania.cl/index.php/magallania/article/view/625

» Prates, L. y Bonomo, M. (2017). Los ambientes acuáticos en arqueología. Arqueología, 23(3), 11-33. https://doi.org/10.34096/arqueologia.t23.n3.4006

» Reyes, M. R. y Svoboda, A. (2016). Un acercamiento a las artes de pesca a partir del análisis de los pesos líticos en el área de los lagos Musters y Colhué Huapi (provincia de Chubut). En F. Mena (Ed.), Arqueología de la Patagonia: de mar a mar (pp. 496-509). Santiago de Chile: Ediciones CIEP/Ñire Negro.

» Reyes, M., Peralta González, S. y Svoboda, A. (2016). Pesos líticos en el área del lago Colhué Huapi (provincia de Chubut): primeros resultados de los análisis tecno-morfológicos y distribucionales. Trabajo presentado en XIX Congreso Nacional de Arqueología Argentina, Tucumán, Argentina.

» Scartascini, F. L. y Cardillo, M. (2009). Explorando la variabilidad métrica y morfológica de las "pesas líticas" recuperadas en el sector norte de la costa del golfo San Matías. En O. M. Palacios, C. Vázquez, T. Palacios y E. Cabanillas (Eds.), Arqueometría latinoamericana: Segundo Congreso Argentino y Primero Latinoamericano (pp. 162-168). Buenos Aires: Comisión Nacional de Energía Atómica - CNEA.

»Schuster, V. (2011). Organización tecnológica de la cerámica de grupos cazadores-recolectores de la costa centro-septentrional de Patagonia (provincia de Chubut, Argentina). (Tesis Doctoral inédita), Universidad Nacional del Centro de la Provincia de Buenos Aires, Argentina.

»Scordo, F., Seitz, C., Zilio, M., Melo, W. D., Piccolo, M. C. y Perillo, G. M. (2017). Evolución de los Recursos Hídricos en el "Bajo de Sarmiento" (Patagonia Extra Andina): Impactos Naturales y Antrópico. Anuário do Instituto de Geociências, 40(2), 106-117.

»Svoboda, A. (2017). Tecnología de pesca en la Patagonia central: un estudio comparativo de los pesos líticos de la costa norte del Chubut y de los lagos Musters y Colhué Huapi. En J. Gómez Otero (Ed.), Libro de resúmenes de las X Jornadas de Arqueología de la Patagonia (pp. 44). Puerto Madryn: Instituto de Diversidad y Evolución Austral.

»Svoboda, A. (2019). Ictioarqueología del sitio Boliche Jeréz 3 (lago Colhué Huapi, Chubut, Argentina): implicaciones para la subsistencia de cazadores-recolectores del Holoceno tardío. Revista de Antropología del Museo de Entre Ríos, 5(1), 54-67. https://ppct.caicyt.gov. ar/index.php/antropmuser/article/view/13708/45454575768269

» Svoboda, A. y Moreno, E. J. (2018). Peces y coipos: zooarqueología del sitio Valle Hermoso 4 (lago Colhué Huapi, Chubut). Revista del Museo de Antropología, 11(1), 85-98. https://doi. org/10.31048/1852.4826.v11.n1.15601 
» Torres, J. E. (2007). ¿Redes o líneas de pesca?: el problema de la asignación morfofuncional de los pesos líticos y sus implicancias en las tácticas de pesca de los grupos del extremo austral de Sudamérica. Magallania, 35(1), 53-70. https://dx.doi.org/10.4067/S071822442007000100004

» Trivi de Mandri, M. y Burry, L. S. (2007). Paleoambientes del Lago Colhué Huapi (Chubut, Argentina) durante el Holoceno reciente. Estudio palinológico. Revista Española de Micropaleontología, 39(3), 205-214. 